\title{
Allgemeiner Anzeiger.
}

\section{Anzeiger der Vereinszeitung.}

\section{Notizen aus der Generalcorrespondenz des Directoriums.}

Se Exo. Hr. Mininter Eichhorn in Berlin: Die Portoverglinstigung fiir den Verein betr. - Kurfürstlich Hessisches Ober-Medicinal-Collegium in Cassel: Mittheilung der dortigen neuen veränderten Arzneitaxe. - Herr Hofapoth. Duff in Kudolstadt: Ueber Angelegenheiten des Kreises Saalfeld. - Die Hrn. Kreisdirectoren Wacken roder in Burgitorf, Becker in Peine, Upmann in Nenenkirchen, Röh $r$ in Crefeld, Freund in Saalfold: Ueber Angelegenheiten der dortigen Kreise. - Hr. Assessor $\mathbf{F}$ aber in Minden: Die Rovision der Rechnung des Vereins betr. - Hr. Ap. Sparkuhl in Andreasberg: Ueber den projectirten Kreis fïr den Ober-Harz. - Hr. Mrdicinalrath Dr. $M$ ïller in Emmerich: Die Verhältnisse des Vereins in dortiger Gegend und den Kreis Medebach betr. - Hr. Postmeister Pothmann in Lemgo: Das Aversum für die Fürstl. Thurnund Taxischen Posten betr. - Hr. Apotheker Brill in Haina: Ueber Gehülfen-Unterstiutzung. - Hr. VicedirectorDr. Ml eur er: Die dortige Postovergiinstigung betr. - $\mathrm{Hr}$. Kreisdirector Griseke in Fisleben: Die Versammlung in Rraunschweig betr. Hr. Vicedirector Dr. Fied Ier in Cassel: Ueber Ausgahen fir die Lesezirkel. - Hr. Apotheker Dr. Riegel in St. Wendel: Ueber die Bildung eines neuen Kreises, - Hr. Apoth. Witzel in Frankenbero: den Kreis Medebach betr.

Dankschreiben für die Ehrenmiigliedschaft des Vereins gingen ein: Von Herrn Vicekanzler Kathold in Rudolstadt; von Herrn Regierungsrath Schwarz und Herrn Medicinalrath Dr. Hankel daselbst.

Dankschreiben fur die von der Hagen - Bucholzschen Stiftung ertheilten Prämien: Von den Herren $S c h m i l t$ in Grimmen, Brendeke in Gittelde, O. Eder in Dresden, Capau derzeit in Warschau.

Gesucho um Unterstutzung: Von den Herren Martin in Driburg, Alberti in Hannover.

Beiträge zum Archiv: Vom Hern Aproth. Ohlert in Miloslaw, vom Hrn. Vicedir. Dr. Meurer in Dresden, voin Hrn. Dr. Holl das., vom Hrn. Kreisdirector Jonas in Eilenburg, Hra. Dr. Bley in Bernburg, Hrn. Professor K a staer in Erlangen, Hr. Pasquier in Lüttich.

\section{An die Herren Mitglieder des Vicedirectoriums} Arusberg und Kreises Medebach inslesondere.

Bei der Verlegung meines Wohnsitzes von Mcdebach nach Rmmerich fuhle ich mich verpflichtet, den verehrten Herrea 
Collegen und Mitgliedern des von mir bisher verwalteten Kreises Medebach ein herzliches Lebewohl zu sagen. Nicht ohne Trauer scheide ich aus lhrer Mitte, geehrte Collegen und Freunde, denn die Freundschaft, mit. welcher Sie mich bei der Verwaltung der Geschäfte des Vereins von Anfarg an stets zu unterstutzen die Güte hatten, habe ich von jeher zu schätzen sewurst und habe namentlich den Herren, welche sich jahrlich auf unseren Kreisversammlungen einfanden, meinen Dank immer herzlich auszudricken mich bemüht. Aber auch in der Ferne soll das freundschaftliche Verhältuifs unter uns fortleben, und wird es mich freuen zu sehen, dafs sich das vereinte Band immer fester knüpfe und so das mit Mühe von mir Gegründete stets erhalten werde, wofür uns Herr College B la Is in Felsberg, der nit Genehmigung ds Oberdirectorii zu meinem Nachfolger designirt wurde, vermöge seiner anerkannten Thätigkeit und liebe fiir die gute Sache, die sicherste Bürgschaft leistet.

Das von mir bisher verwaltete Vicedirectorium Arnsberg erleidet nun mit dem 1. Januar 1842 folgende Veränderungen:

1. Die Leitung des Vicedirectoriums ubernimmt Herr College Posthoff in Siegen.

II. Die Geschäfte des Kreises Medebach wird Herr College Bafs in Felsberg ubernehmen und werden zu diesem Kreise folgende Mitglieder gehören:

1) Herr Apotheker Blafs in Felsberg, Kreisdirector.

(3) $D$ Bender in Spangenberg.

3) $»$ Göllner in Wildungen.

4) * * Müll er daselbst.

5) $\quad$ Henke in Arolsen.

6) * $\quad$ Schmidt in Mengeringhausen.

7) $\rightarrow \quad$ Iskenius in Marsberg.

8) $\diamond \quad$ Wald schmidt in Sachsenhausen.

9) * Kunkel in Corbach.

10) " Doctor Ruve daselbst.

11) "Apotheker Heinzerling in Voehle.

13) * Wirth in Sachsenberg.

13) " Doctor Hartwig in Frankenberg.

14) * Apotheker Witzel daselbst.

15) * Brill in Haina.

16) * Wangemann in Fauschenberg.

17) " Köchling in Rosenthal.

18),$\quad \mathbf{K u m m e l}$ in Miunchhausen.

19) $\Rightarrow$ Kindervatter in Wetter.

III. Der Kreis Siegen, unter Leitung des Vicedirectors Apothekers Posth off, wirl, dat in demselben bisher nur wenige Mitglieder waren, durch mehre aus dem Kreise Medebach vermehrt und folgende Mitglieder haben:

i) Hor Apotheker Posth off in Siegen, Vicedirector.

2) " Musset daselbst."

3) * Grofsmann in Battenberg.

4) * Schue in Biedenkopf.

5) " $\quad$ lang in Gladenbach.

c) " Hoeseler in Winterberg.

7) * Niemann in Bigge.

8) * Hillenkamp in Brilon. 
9) Herr Apotheker Reinige in Lippstadt.

10),$\quad$ Wrede in Freudenberg.

11) $:$ Wisthoff in 0lpe.

12) \# Hollmann in Plettenberg.

13) , Neuhoff in Hilchenbach.

14) $\quad$ Kraemer in Kirchen.

15) : Schütz in Berleburg.

16) Bottrich in Schmallenberg.

1V. Der Kreis Arnsberg unter Leitung des Herrn Kreikdirectors Apotheker Müller in Arnsberg bleibt unverändert.

Emmerich, den 14. Navemb. 1841. Dr. Müller.

\section{Den Kreis Meiningen betreffend.}

Hr. Medicinalassessor $\mathrm{Jahn}$ in Meiningen hat wiederholt gewünscht, die von ihm so trefflich geleitete Verwaltung des Kreises Meiningen aufzugeben, weil anderweitige iiberhäufte Arbeiten ihn linderten, der Verwaltung ferner die zur Förderung der guten Sache nothwendige Zeit zu widmen. Das Directorium hat diesem $W$ unsche endlich nachzugeben sich genöthigt finden miussen. Es geschieht aber nicht, ohne dem Hrn. Collegren Jahn für seine dem Vereine so vielfach geleisteto schöne Mitwirkung öffentlich den herzlichsten Dank darzubriugen.

Den verehrten Herren Mitgliedern des Kreises Meiningen machen wir hiermit zugleich die weitere Anzeige, dafs unser verehrter College, Hr. Hofapotheker Löhlein in Coburg dio Giite gehabt hat, die Verwaltung des Kreises zu übernehmen, dafs dieser Kreis nun als der Kreis Coburg aufgefuhrt wird und dafs wir die verehrten Herren Mitglieder desselben ersuchen, in Vereinsangelegenheiten an Hrn. Kreisdirector Löhlein gefulligst sich zu wenden.

Der Oberdirector des Vereins. Dr. Brandes.

\section{Weitere Verbreitung des Vereins.}

Hr. Apotheker Sparkuhl in Andreasberg wird einen neuen Kreis am Oberkarze begrianden, zu weIchem noch einige Collegen aus dem Kreise Eimbeck, der besseren Verbindung wegen, hinzutreten werden.

Hr. Medicinalrath Dr. Müller in Emmerich ist mit der Bildung eines neuen Kreises in dortiger Gegend thätig beschäftigt.

Hr. Apotheker Dr. Riegel in St. Wendel hat daselbst einen neuen Kreis begründet. Sobald die Sache definitiv geordnet ist, wird das Nälhere dariber mitgetheilt werden.

\section{Handelsnotizen.}

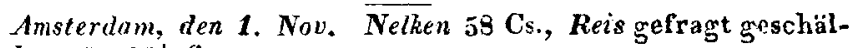
ter Java $9-10 \frac{1}{\frac{1}{3}}$ fl.

- den 8. Nov. Von Surinam-Cacao sind einzelne kleine Parthien zu verschiedenen Pruisen verkauft worden; von Ma- 
ranhan und Guajaquil ist in erster Hand nichts vorräthig. Guajaquil wird mit 24 Cs., Maranhon mit 27 Cs., Surinam mit $14-23 \mathrm{Cs}$. notirt.

- den 15. Nov. Malagaer Rosinen $12 \mathrm{fl}$., Muscateller $21 \mathrm{fl}$., Smyrnaer $7-9 \mathrm{fl}$., Samos $8 \mathrm{fl}$, schwarze $6 \frac{1}{2} \mathrm{fl}$. Feigen $8 \frac{1}{3} \mathrm{fl}$.

Bremen, den 15. Nov. Der herannahende Winter wird uns nun bald die Zufuhren zur See abschneiden, obgleich es wïnschenswerth bleibt, dafs aus der Mittelländischen See uns noch Einiges von der neuen Mandel-Erndte zugehen möchte, was auf die Preise bedeutenden Einflufs haben würde. Anch von $O e l$ war die Erndte gut, doch ist der Bedarf davon, besonders für Fabriken, so grofs, dafs die Preise nicht bedeutend sinken können. Die Erndte der Rosinen und Corinthen war sehr reichhaltig, und besonders letztere werden bedeutend in Preise sinken. Die Ereignisse in China haben noch zu keinem Resultat gefuhrt, und herrscht noch immer dieselbe Ungewifsheit. Die wenigen Zufuhren von rohem Campher legten sich so hoch her, dals dieselben den Raffineurs Verlust brachten, da der raffinirte noch $z u$ billig steht. Wir durfen eine Steigerung davon gewifs erwarten. Mit Rhabarber geht es leider sehr unglücklich, da eine kleine Zufuhr von 300 Kisten aus China nach Amerika in Seo ganz verungluickt ist, und man sonst nichts erwartet. Aloe bleibt preishaltend, da die Zufuhren sehr mälsig waren. Cacao ziemlich fest im Preise, eine gesunde Guajaquil kann empfohlen werden, Brasil ohne Vorrath, rothe Bahia, schön für's Auge, findet wegen ihres bitteren Geschmacks wenig Liebhaberei. Caryophill.arom, sind seit der letzten holländ. Auction billiger. China und deren Salze ganz ohne Frage. In Gummatib gute Auswahl, nur Elemi fehlt. Schellack sehr niedrig im Preise, verdient Beachtung. Fucus amylaceus, wovon erst wenig in Handel gebracht ist, möchte in der Folge weit mehr berücksichtigt werden. Nieses Moos ibertrifft das Caragheen, und enthält keine salzige Stoffe oder Jod, weshalb es nicht bitter schmeckt, und für Kinder und säugende Mutter stets mit grolsem Vortheil gebraucht werden kann.

Die Zufuhren von Sassaparille waren sehr bedeutend, dagegen auch der Abzug nach Rulsland sehr stark. Es findet sich aber viele sehr ordinaire Waare dabei, welche selbst zu sehr niedrigen Preisen nicht zu empfehlen ist. Von Serpentaria trafen mohre Zufuhren ein, auch etwas Senega. Sem. Lycopod. bedeutend billiger. Die Essenzen halten sich hoch, besonders ist $O l$. Bergamo fiir England weggekauft. $O l$. Terebinth ist in Frankreich im Preise gestiegen. Von Colophonium waren dio Zufuhren aus Amerika sehr bedeutend, es fehlte nicht an Speculanten, welche dasselbe auf's Lager nahmen. Im Uebrigen ohne bedeutende Veränderungen.

Cephalonia, den 12. Oct. Im Allgemeinen mögen bis jetzt 6 Mill. Pfd. Corinthen veräulsert sein.

Hamburg, den 3. Nov. Gewürze wenig gefragt, besonders Nelken, sülse Berb. Mandeln sind im Preise gesunken, Sicilische ihrer Seltenheit wegen höher gegangen.

- den 12. Nov. Unsere Vorräthe von billern Mandeln sind bis auf einigre Kleinigkeiten geräumt, für die hohe Preise 
gefordert werden. Malagaer Mandeln, etwas kleiner als Valenzer sind zu 59 Mk. bedungen. Beste Muscatnüsse $35-36 \mathrm{Mk}$, Macisblumen $29 \frac{1}{2}$ Mk. Es gingen 50,000 Pfd. Ostind. Ingwer zu 1\% Mk. 2 sh. Nelken sind noch im Preise gresunken.

- den 19. Nov. Cassa lignea stark begehrt $12 \frac{3}{1} \mathrm{sh}$.

Leipzig, den 6. Nov, Rüböl 15 Thlr., Leinöl 13 Thlr., Mohnol $19-19 \frac{1}{2}$ Thlr.

- den 13. Nov. Rüböl ist auf $15 \frac{1}{4}$ Thlr. gekommen, Leinöl

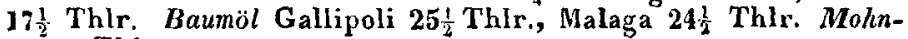
ol 19 Thlr.

London, den 12. Nov. Congo-Thee begehrt 1 sh. 16 d. Cacao Westind. $1-2$ sh. im Preise gewichen, ferner roth. Trinidad $48-49$ sh., guter $44-46$ sh., mittel $41-43$, ord. $37-$ 41 sh. Calcutta Salpeter ord. 26 sh., guter $27-29$ sh. Seit den Nachrichten von Calcutta, welche die Indig-Erndte auf das grofse Quantum von 140 - 145,000 Maunds rechnen, ist der Preis etwas gesunken. Cochenille begehrt, Honduras mittel-silber 3 sh. 7 d. -4 sh. 1 d.

- den 3. Dec. Die Preise von Westind. Cacao haben sich vollkominen behauptet.

Neapel, den 21. Nov. Die Oele haben einen neuen Abschlag erlitten; farbiges Gallipoli $39 \mathrm{D}$, künftiges $27 \mathrm{D} .70 \mathrm{Gr}$.

- den 28. Nov. Nach den neuesten Berichten aus Sicilien, wie auch aus Calabrien und der Provinz Bari, scheint der Ertrag der Oel-Erndte ergiebig.

Stetlin, den 12. Nov. In Rüböl wenig Umsatz, $14 \frac{1}{2}$ Thlr., Leinöl $11_{4}^{1}$ Thlr., Dotteräl $12 \frac{1}{2}$ Thlr., Palmöl 12⿺ Thlr., Südseethran 9! Thlr., heller Robbenthran 12 Thlr., brauner Berger Leberthran $21 \frac{1}{2}$ Thlr., blanker 25 Thlr.

\section{Anzeige fïr Freunde der Botanik.}

Hr. Gardin, Apothekenbesitzer in Charlestown, beabsichtigt die Flora von Nordamerika in Centurien zu liefern, wenn sich dazu eine Anzahl Abnehmer finden sollten. Die Centurie gut getrockneter Pflanzen wird bei vorausgegangener Bestellung zu sechs Thlr. Preufs. Cour. ab Blankenburg am Harz goliefert, die Zahlung geschieht gegen Empfangnahme der Pflanzen. - Fur die Bestimmung und Vertheilung an die resp. Interessenten sorgt der Unterzeichnete, und bittet derselbe um baldige Aufträge portofrei.

Blankenburg a. Harz, Decbr. 1841.

Ernst Ha inpe.
Noch sind mehre Fascikel gut getrocknete, seltene Pflanzen der Schweiz à 150 Arten für 1 Louisd'or bei mir zu haben. Derselbe.

\section{Dienstgesuche.}

Fiir einen sehr zu empfehlenden Gehiilfen, der sofort odor zu nächsten Ostern eintreten kann, suche ich eine Stelle, und werde das Nähere auf gefallige Anfrage sofort mittheilen.

Der Apotheker Kittel

zu Dornum in Ostfriesland. 


\section{Dienstanträge.}

Ein der deutschen und polnischen Sprache mächtiger junger Mann findet als Gehülfe in der Musenber'schen Apotheke zu Ostrowo, wenn er empfehlende Zeugnisse aufzuweisen, gegen anständiges Gehalt zum 1. April 1842 ein Unterkommen. Briefa werden an Herrn Provisor $G$ e is 1 e $r$ in Ostrowo erbeten.

A. Lipowitz,

Kreisdirector des Apothekervereins in Norddeutschland, in Lissa.

Eine Anzahl zum Theil sehr gut empfohlener alterer und jüngerer Pharmaceuton, wïnscht zum Antritt auf 0 stern vacante Gehülfenstellen nach Befinden in grörsern oder kleinern Oficinen zu übernehmen.

Erfurt in December 1841.

Eduard Grefsler.

\section{Nachweisung zur Besetzung vacanter Apotheker- gehülfenstellen.}

Mehrfachen Anfragen zu geniigen, mache ich meinen Hrn. Collegen bekannt, dafs auch ich wie mein Vorfahrer, Herr Gress l er, jetzt dem Kaufmannstand in Erfurt angehörig, die Besetzung vacanter Gehüfenstellen unter folgenden Bedingungen besorge:

Lie Herren Collegen, die eine erledigte Stelle durch meine Vermittlung besetzt sehen wollen, ersuche ich hei zeitiger Anmeldung un genaue Angabe aller der Erfordernisse die ein zur vacanten Stelle sich eignender Gehülfe besitzen soll, um dann die $W$ ahl durch passende $Z$ uweisung ihnen erleichtern zu können.

Alle Herren Gehülen aber, welche ein Engagement zu erhalten winschen, ersuche ich, ein Curriculum vilae, beglaubigte Abschrift ihrer Zeugnisse, so wie Angabe der zu stellenden Anforderungen einzusenden, worauf ich gecignete Nachweisung von Vacanzen anzeigen und dieselben bei den betreffenden Herren Collegen empfehlen werde.

Nur dann, wenn durch mich ein Fingagement bewirkt, so ist ein Honorar von 2 Thlr. Preufs. Cour. zu entrichten, bei Nachweisungen aber, die ohne Erfolg ghlieben, ist für meine Mühe kein Theil mir Entschadigung schuldig.

Sofortige Anzejge von Anuahme oder anderweitiger Besetzung einer Stelle und portofreie Zusendungen in diesen Angelegenheiten werden erbeten.

Durch diese Einrichtung glaube ich meinen Herren Collegen uberflïssigen Zeitaufwand, den Herren Gehiulfen aber dio unnöthigen Kosten leerer Nachweisung ersparen zu können.

Saalfeld in Thüringen, don 4. Dec. 1841.

N. F reund, Apotheker. 